\title{
Statyba
}

\section{DUAL MATHEMATICAL MODELS OF ELASTOPLASTIC STRUCTURES ANALYSIS PROBLEM ESTIMATING DISCONTINUITY OF DISPLACEMENTS}

\section{S. Kalanta}

To cite this article: S. Kalanta (1995) DUAL MATHEMATICAL MODELS OF ELASTOPLASTIC STRUCTURES ANALYSIS PROBLEM ESTIMATING DISCONTINUITY OF DISPLACEMENTS, Statyba, 1:3, 5-29, DOI: 10.1080/13921525.1995.10531517

To link to this article: https://doi.org/10.1080/13921525.1995.10531517

Published online: 26 Jul 2012.

Submit your article to this journal $\llbracket$

Џ Article views: 58 
ISSN 1392-1525. STATYBA - BUILDING CONSTRUCTION - CTPOИTEЛЬCTBO, 1995, Nr. 3(3)

Statybinè mechanika

\section{ДВОЙСТВЕННЫЕ МАТЕМАТИЧЕСКИЕ МОДЕЛИ ЗАДАЧ АНАЛИЗА УПРУГОПЛАСТИЧЕСКИХ КОНСТРУКЦИЙ С РАЗРЫВНЫМИ ПОЛЯМИ ПЕРЕМЕЩЕНИЙ}

\section{C. Каланта}

\section{1. Введение}

Рассматриваются двойственные конечноэлементные постановки задачи анализа напряженно-деформированного состояния (НДС) строительных конструкций при наличии пластических деформаций. Исследования основаны на физической модели идеально упругопластического материала, теория пластического течения и общепринятых при этом допущениях [1]. Двойственные математические модели этой задачи представлены в работе [2] для стержневых систем и в [1] для обобщенной дискретной конструкции при разных видах нагружения, нелинейных и линейных условиях текучести. Задачи двойственного анализа НДС упругопластических изгибаемъх пластинок в работах $[3,4]$ формулируются и решаются с применением метода конечных элементов. Ках известно, пластическое разрушение тонких пластинок и оболочек обычно происходит из-за образования так называемых линейных пластических шарниров (линий разрушения), где имеют место разрывы перемещений и гроисходит диссипация энергии. Однако в [3, 4] и других работах, где двойственные математические модели задач анализа строятся с применением равновесных конечных элементов (РКЭ), не предусматривается возможность образования таких шарниров, т. е. негласно принимается предположение о гладкости функций перемещений. Очевидно, что такие математические модели не могут с достаточной точностью моделировать реальное деформированное состояние пластинок и оболочек, особенно близкое $\mathbf{k}$ предельному. Двойственные математические модели задач пределного равновесия для конечноэлементных систем с учетом возможных разрывов скоростей перемещений рассматривались в [5]. Как продолжение исследований $[5,6]$ по совершенствованию конечноэлементных математических моделей в настоящей работе строятся двойственные постановки задачи анализа НДС упругопластических конструкций, в которых учитываются разрывы полей перемещений в местах образования пластических шарниров и в 
поверхностях начальных разрывов (трещинах), если они существуют. В качестве объекта исследований выбрана тонкостенная конструкция. Однако построенные конечноэлементные математические модели распространяются как на стержневые системы, так и на трехмерные тела.

\section{2. Общие математические модели задачи}

Тонкостенная конструкция, геометрические параметры и предельные усилия $\mathbf{S}_{0}(\mathbf{x})$ которой заданы, подвергается действию внешней нагрузки $\mathbf{F}(\mathbf{x})$, начальных деформаций $\Theta_{0}(\mathbf{x})$ и перемещений $\mathbf{u}_{0}(\mathbf{x})$. Пусть ее срединная поверхность разделена на $s$ конечных элементов площади $A_{k}$ и между ними могут быть разрывы некоторнх перемещений.

Искомое НДС $k$-го элемента описывается вектор-функциями усилий или напряжений $\mathbf{S}_{k}(\mathbf{x})=\mathbf{S}_{e k}(\mathbf{x})+\mathbf{S}_{r k}(\mathbf{x})$, перемещений $\mathbf{u}_{k}(\mathbf{x})=\mathbf{u}_{e k}(\mathbf{x})+\mathbf{u}_{r k}(\mathbf{x})$ и деформаций $\Theta_{k}(\mathbf{x})=\Theta_{e k}(\mathbf{x})+\Theta_{r k}(\mathbf{x})+\Theta_{0 k}(\mathbf{x})$. Здесь индексом $e$ обозначены параметры упругого расчета, а индексом $r$ - остаточные параметры. Функции, относяциеся к смежным конечным элементам, разделенным линией $L_{t}$, обозначаются знаками “+" и " - ". Для краткости изложения материала далее все вектор-функции обозначаются знаком “ “ над соответствуюшим символом, а вектор координат $\mathbf{x}$ опускается, т. е. вводятся обозначения $\mathbf{S}_{k}(\mathbf{x})=\widetilde{\mathbf{s}}_{k}, \mathbf{u}_{k}(\mathbf{x})=\widetilde{\mathbf{u}}_{k}$ и другие. Это делается для того, чтобы различить вектор-функции от векторов узловых значений этих функций.

В качестве неизвестных задачи могут быть приняты суммарные или остаточные усилия и перемещения, так как упругие составляюпие напряженнодеформированного состояния могут быть определены предварительным упругим расчетом конструктии.

\section{1. Задача анализа суммарньх усилий и перемешений}

Математическая модель статической постановки задачи строится на основе экстремального принципа о минимуме полной дополнительной энергии:

из всех статически допустимых полей усилий действительным является то поле, которому отвечает минимальное значение полной дополнительной энергии.

Множество статически допустимых векторов усилий $\widetilde{\mathbf{S}}_{k}$ описывается уравнениями равновесия внутри элементов

$$
[\mathbf{A}] \widetilde{\mathbf{S}}_{k}=\widetilde{\mathbf{F}}_{k} \quad \in A_{k},
$$

условиями сопряжения 


$$
\begin{gathered}
{\left[A_{t}\right] \widetilde{\mathbf{S}}_{t}^{+}-\widetilde{\mathbf{S}}_{\mathfrak{v}}^{+}=\mathbf{0} \quad \in L_{t}^{+}, \quad-\left[A_{t}\right] \widetilde{\mathbf{S}}_{t}^{-}-\widetilde{\mathbf{S}}_{\mathfrak{v}}^{-}=\mathbf{0} \in L_{t}^{-},} \\
\widetilde{\mathbf{S}}_{v t}^{+}+\widetilde{\mathbf{S}}_{\mathfrak{v}}^{-}=\mathbf{0} \quad \in L_{t},
\end{gathered}
$$

статическими граничными условиями

$$
\left[A_{L}\right] \widetilde{\mathbf{S}}=\widetilde{\mathbf{F}} \quad \in L_{f}
$$

и условиями текучести

$$
\begin{gathered}
\mathbf{f}\left(\widetilde{\mathbf{S}}_{k}\right) \leq \mathbf{f}_{0}\left(\widetilde{S}_{0 k}\right) \quad \in A_{k}, \\
\mathbf{f}_{t}\left(\widetilde{\mathbf{S}}_{v t}^{+}\right) \leq \mathbf{f}_{0 t}\left(\widetilde{S}_{0 t}^{+}\right) \in L_{t}^{+}, \quad \mathbf{f}_{t}\left(\widetilde{\mathbf{S}}_{v t}^{-}\right) \leq \mathbf{f}_{0 t}\left(\widetilde{S}_{0 t}^{-}\right) \in L_{t}^{-} ; \\
k=1,2, \ldots, s, \quad t=1,2, \ldots, z,
\end{gathered}
$$

где $[\mathrm{A}],\left[A_{t}\right]$ - операторы дифференциальных уравнений равновесия конструкции и статических условий сопряжения между элементами; $\widetilde{S}_{0 k}-$ заданная функщия предельного усилия $k$-го элемента; $s$ - число конечных элементов; $\mathrm{z}$ - число поверхностей раздела элементов, для которых составляются условия сопряжения и условия текучести (5).

Тогда статическая постановка задачи представляется следующей математической моделью:

$$
\sum_{k} \frac{1}{2} \int_{A_{k}} \widetilde{\mathbf{s}}_{k}^{T}\left[d_{k}\right] \widetilde{\mathbf{S}}_{k} d A+\sum_{k} \int_{A_{k}} \widetilde{\mathbf{s}}_{k}^{T} \widetilde{\Theta}_{0 k} d A-\int_{L_{u}} \widetilde{\mathbf{u}}_{0}^{T}\left[A_{L}\right] \widetilde{\mathbf{S}} d L \rightarrow \text { min }
$$

при условиях

$$
\begin{aligned}
& \mathbf{f}\left(\widetilde{\mathbf{S}}_{k}\right) \leq \mathbf{f}_{0}\left(\widetilde{S}_{0 k}\right), \quad[\mathrm{A}] \widetilde{\mathbf{S}}_{k}=\widetilde{\mathbf{F}}_{k} \quad \in A_{k}, \\
& \mathbf{f}_{t}\left(\widetilde{\mathbf{S}}_{v t}^{+}\right) \leq \mathbf{f}_{0 t}\left(\widetilde{S}_{0 t}^{+}\right), \quad\left[A_{t}\right] \widetilde{\mathbf{S}}_{t}^{+}-\widetilde{\mathbf{S}}_{\mathfrak{v}}^{+}=\mathbf{0} \quad \in L_{t}^{+} ; \\
& \mathbf{f}_{t}\left(\widetilde{\mathbf{S}}_{v t}^{-}\right) \leq \mathbf{f}_{0 t}\left(\widetilde{S}_{0 t}^{-}\right), \quad-\left[A_{t}\right] \widetilde{\mathbf{S}}_{t}^{-}-\widetilde{\mathbf{S}}_{v t}^{-}=\mathbf{0} \quad \in L_{t}^{-} ; \\
& \widetilde{\mathbf{S}}_{v^{t}}^{+}+\widetilde{\mathbf{S}}_{\boldsymbol{v} t}^{-}=\mathbf{0} \in L_{t}, \quad\left[A_{L}\right] \widetilde{\mathbf{S}}=\widetilde{\mathbf{F}} \in L_{f} ; \\
& k=1,2, \ldots, s ; \quad t=1,2, \ldots, z .
\end{aligned}
$$

Здесь $\left[d_{k}\right]$ - матрища податливости элементарного элемента конструкции. Задача (6) позволяет определить распределение действительных усилий. Ее функция цели имеет физический смысл полной цополнительной энергии. При отсутствии начальных деформаций она сводится $\mathrm{k}$ принципу Хара-Кармана [7]. В случае неограниченно болыших предельных усилий $\left(S_{0}=\infty\right)$ она отвечает вариационному принципу Кастильян.

Математическая модель двойственной кинематической постановки задачи строится по методу множителей Лагранжа и получает такой вид: 


$$
\begin{aligned}
& \int_{L_{f}} \widetilde{\mathbf{u}}^{T} \widetilde{\mathbf{F}} d L+\sum_{k} \int_{A_{k}} \widetilde{\mathbf{u}}_{k}^{T} \widetilde{\mathbf{F}}_{k} d A-\sum_{k} \frac{1}{2} \int_{A_{k}} \widetilde{\mathbf{s}}_{k}^{T}\left[d_{k}\right] \widetilde{\mathbf{S}}_{k} d A-\sum_{k} \int_{A_{k}} \tilde{\lambda}_{k}^{T}\left[f\left(\widetilde{\mathbf{S}}_{k}\right)\right] \widetilde{\mathbf{S}}_{k} d A-
\end{aligned}
$$

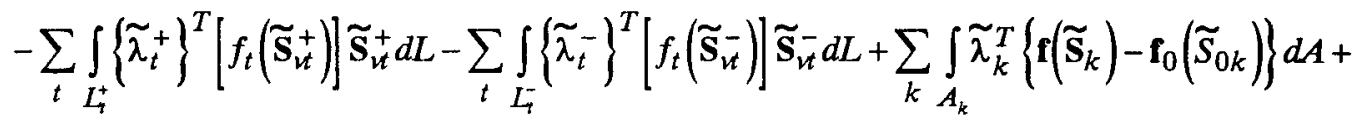

$$
\begin{aligned}
& +\sum_{t} \int_{L_{t}^{+}}\left\{\tilde{\lambda}_{t}^{+}\right\}^{T}\left\{\mathbf{f}_{t}\left(\widetilde{\mathbf{S}}_{v t}^{+}\right)-\mathbf{f}_{0 t}\left(\tilde{S}_{0 t}^{+}\right)\right\} d L+\sum_{t} \int_{L_{t}^{-}}\left\{\tilde{\lambda}_{t}^{-}\right\}^{T}\left\{\mathbf{f}_{t}\left(\widetilde{\mathbf{S}}_{v t}^{-}\right)-\mathbf{f}_{0 t}\left(\tilde{S}_{0 t}^{-}\right)\right\} d L \rightarrow \max
\end{aligned}
$$

при условиях

$$
\begin{gathered}
{\left[d_{k}\right] \widetilde{\mathbf{s}}_{k}+\Theta_{0 k}+\left[f\left(\widetilde{\mathbf{s}}_{k}\right)\right]^{T} \tilde{\lambda}_{k}-[\mathbf{A}]^{T} \widetilde{\mathbf{u}}_{k}=\mathbf{0}, \quad \tilde{\lambda}_{k} \geq \mathbf{0} \in A_{k} ;} \\
{\left[f_{t}\left(\widetilde{\mathbf{s}}_{u}^{+}\right)\right]^{T} \tilde{\lambda}_{t}^{+}-\widetilde{\mathbf{u}}_{t}+\widetilde{\mathbf{u}}_{t}^{+}=\mathbf{0}, \quad \tilde{\lambda}_{t}^{+} \geq \mathbf{0} \in L_{t}^{+} ;} \\
{\left[f_{t}\left(\widetilde{\mathbf{s}}_{u}^{-}\right)\right]^{T} \tilde{\lambda}_{t}^{-}-\widetilde{\mathbf{u}}_{t}+\widetilde{\mathbf{u}}_{t}^{-}=\mathbf{0}, \quad \tilde{\lambda}_{t}^{-} \geq \mathbf{0} \in L_{t}^{-} ;} \\
{\left[A_{L}\right]^{T}\left(\tilde{\mathbf{u}}-\widetilde{\mathbf{u}}_{0}\right)=\mathbf{0} \in L_{u} ; k=1,2, \ldots, s ; t=1,2, \ldots, z .}
\end{gathered}
$$

Здесь $\tilde{\lambda}_{k}, \tilde{\lambda}_{t}^{+}, \tilde{\lambda}_{t}^{-},\left[f\left(\widetilde{\mathbf{S}}_{k}\right)\right],\left[f_{t}\left(\widetilde{\mathbf{S}}_{v^{t}}^{+}\right)\right],\left[f_{t}\left(\widetilde{\mathbf{S}}_{v^{t}}^{-}\right)\right]-$функщии пластических множителей и матрицы градиентов функций текучести $k$-го элемента и $t$-й линии раздела элементов. Неизвестными являются вектор-функщии усилий, перемещений и пластических множителей. Выясним физический смысл этой задачи.

Три последних члена целевой функции для оптимального решения задачи равны нулю. Третий и четвертый интегралы выражают энергию деформаций в конечных элементах, а пятый и шестой интегралы - диссипативную энергию в линейных пластических шарнирах. Поэтому функция цели, умноженная на -1 , имеет смысл полной энергия деформаций. Ограничения-равенства представляют собой уравнения геометрической совместности конечных элементов, межэлементной совместности и кинематические граничные условия на опорном контуре $L_{u}$. Вместе с ограничениями знака пластических множителей они описывают множество кинематически допустимых вектор-функций перемещений. Таким образом, задача (7) отвечает следующему экстремальному принципу:

из всех кинематически допустимых полей перемещений действительным является то поле, для которого полная энергия деформаций конструкции минимальна.

Предполагая $\widetilde{\mathbf{S}}_{v t}=\widetilde{\mathbf{S}}_{v t}^{+}=-\widetilde{\mathbf{S}}_{v t}^{-}$и преобразуя условия сопряжения (2) к виду

$$
\left[A_{t}\right] \widetilde{\mathbf{S}}_{t}^{+}-\widetilde{\mathbf{S}}_{v t}=\mathbf{0}, \quad-\left[A_{t}\right] \widetilde{\mathbf{S}}_{t}^{-}+\widetilde{\mathbf{S}}_{v t}=\mathbf{0} \quad \in L_{t},
$$

в математических моделях задачи можно уменьшить число неизвестных и уравнений. Получается следующая пара двойственных модифицированных задач математического программирования: 
a) статическая постановка -

$$
\sum_{k} \frac{1}{2} \int_{A_{k}} \widetilde{\mathbf{S}}_{k}^{T}\left[d_{k}\right] \widetilde{\mathbf{S}}_{k} d A+\sum_{k} \int_{A_{k}} \widetilde{\mathbf{S}}_{k}^{T} \widetilde{\Theta}_{0 k} d A-\int_{L_{u}} \widetilde{\mathbf{u}}_{0}^{T}\left[A_{L}\right] \widetilde{\mathbf{S}} d L \rightarrow \min
$$

при условиях

$$
\begin{array}{llll}
\mathbf{f}\left(\widetilde{\mathbf{S}}_{k}\right) \leq \mathbf{f}_{0}\left(\widetilde{S}_{0 k}\right) & \in A_{k}, & \mathbf{f}_{t}\left(\widetilde{\mathbf{S}}_{\mathrm{v} t}\right) \leq \mathbf{f}_{0 t}\left(\widetilde{S}_{0 t}\right) & \in L_{t}, \\
{[\mathbf{A}] \widetilde{\mathbf{S}}_{k}=\widetilde{\mathbf{F}}_{k} \quad \in A_{k},} & {\left[A_{L}\right] \widetilde{\mathbf{S}}=\widetilde{\mathbf{F}}} & \in L_{f}, \\
{\left[A_{t}\right] \widetilde{\mathbf{S}}_{t}^{+}-\widetilde{\mathbf{S}}_{\mathbf{v} t}=\mathbf{0},} & -\left[A_{t}\right] \widetilde{\mathbf{S}}_{t}^{-}+\widetilde{\mathbf{S}}_{\mathrm{v} t}=\mathbf{0} & \in L_{t} ; \\
k=1,2, \ldots, s ; & t=1,2, \ldots, z .
\end{array}
$$

б) кинематическая постановка -

$$
\begin{aligned}
& \int_{L_{f}} \widetilde{\mathbf{u}}^{T} \widetilde{\mathbf{F}} d L+\sum_{k} \int_{A_{k}} \widetilde{\mathbf{u}}_{k}^{T} \widetilde{\mathbf{F}}_{k} d A-\sum_{k} \frac{1}{2} \int_{A_{k}} \widetilde{\mathbf{S}}_{k}^{T}\left[d_{k}\right] \widetilde{\mathbf{S}}_{k} d A-\sum_{k} \int_{A_{k}} \tilde{\lambda}_{k}^{T}\left[f\left(\widetilde{\mathbf{S}}_{k}\right)\right] \mathbf{S}_{k} d A-\sum_{t} \int_{L_{i}} \tilde{\lambda}_{t}^{T}\left[f_{t}\left(\widetilde{\mathbf{S}}_{\mathfrak{v}}\right)\right] \widetilde{\mathbf{S}}_{\mathfrak{k}} d L+ \\
& +\sum_{k} \int_{A_{k}} \tilde{\lambda}_{k}^{T}\left\{\mathbf{f}\left(\widetilde{\mathbf{S}}_{k}\right)-\mathbf{f}_{0}\left(\widetilde{S}_{0 k}\right)\right\} d A+\sum_{t} \int_{L_{t}} \tilde{\lambda}_{t}^{T}\left\{\mathbf{f}_{t}\left(\widetilde{\mathbf{S}}_{\mathfrak{t} t}\right)-\mathbf{f}_{0 t}\left(\widetilde{S}_{0 t}\right)\right\} d L \rightarrow \max
\end{aligned}
$$

при условиях

$$
\begin{gathered}
{\left[d_{k}\right] \widetilde{\mathbf{s}}_{k}+\widetilde{\Theta}_{0 k}+\left[f\left(\widetilde{\mathbf{s}}_{k}\right)\right]^{T} \tilde{\lambda}_{k}-[\mathbf{A}]^{T} \widetilde{\mathbf{u}}_{k}=\mathbf{0}, \quad \tilde{\lambda}_{k} \geq \mathbf{0} \in A_{k} ;} \\
{\left[f_{t}\left(\widetilde{\mathbf{s}}_{u t}\right)\right]^{T} \tilde{\lambda}_{t}-\widetilde{\mathbf{u}}_{t}^{-}+\widetilde{\mathbf{u}}_{t}^{+}=\mathbf{0}, \quad \tilde{\lambda}_{t} \geq \mathbf{0} \in L_{t} ;} \\
{\left[A_{L}\right]^{T}\left(\widetilde{\mathbf{u}}-\widetilde{\mathbf{u}}_{0}\right)=\mathbf{0} \in L_{u} ; k=1,2, \ldots, s ; t=1,2, \ldots, z .}
\end{gathered}
$$

Если $\tilde{\lambda}_{t}^{+}=0$ и $\tilde{\lambda}_{t}^{-}=0$ или $\tilde{\lambda}_{t}=0$, т. е. в линии $L_{t}$ пластические деформации отсутствуют, то геометрические уравнения выражают требование неразрывности перемещений

$$
\widetilde{\mathbf{u}}_{t}^{+}-\widetilde{\mathbf{u}}_{t}^{-}=\mathbf{0} \in L_{t} .
$$

Поэтому математические модели (7) и (9) обеспечивают непрерывность перемещений в упругих зонах конструкции. В отличие от (6) и (8) они позволяют определить все параметры НДС. В случае неограниченной линейной упругости они отвечают вариационному принципу Лагранжа.

Если существуют поверхности начальных разрывов (трещины), то они не включаются в число $z$ поверхностей раздела конечных элементов и условия (2), (5), а также условия межэлементной геометрической совместности для них не составляются. Однако эти поверхности включаются в граничную область $L_{f}$, для которой составляются статические граничные условия (3). 


\section{2. Задача анализа остаточньх усилий и перемещеший}

Если предварительным расчетом определить параметры упругого НДС, то простой заменой переменных задачи (6) - (9) можно преобразовать в тождественные задачи анализа остаточных параметров НдС. Математическая модель задачи определения остаточных усилий строится преобразованием задачи (8).

Множество статически возможных векторов $\widetilde{\mathbf{s}}_{r k}$ описывается уравнениями равновесия

$$
\begin{array}{lccc}
{[\mathbf{A}] \widetilde{\mathbf{S}}_{r k}=\mathbf{0}} & \in A_{k}, & {\left[A_{L}\right] \widetilde{\mathbf{S}}_{r}=\mathbf{0}} & \in L_{f}, \\
{\left[A_{t}\right] \widetilde{\mathbf{S}}_{r t}^{+}-\widetilde{\mathbf{S}}_{r, k t}=\mathbf{0},} & -\left[A_{t}\right] \widetilde{\mathbf{S}}_{r t}^{-}+\widetilde{\mathbf{S}}_{r, k t}=\mathbf{0} & \in L_{t} .
\end{array}
$$

Функционал дополнительной энергии с учетом

$$
\left[d_{k}\right] \widetilde{\mathbf{S}}_{e k}-[\mathbf{A}]^{T} \widetilde{\mathbf{u}}_{e k}=-\widetilde{\Theta}_{0 k} \quad \in A_{k}
$$

и

$$
\begin{aligned}
& \sum_{k} \int_{A_{k}} \widetilde{\mathbf{S}}_{r k}^{T}\left[d_{k}\right] \widetilde{\mathbf{S}}_{r k} d A=\sum_{k} \int_{A_{k}} \widetilde{\mathbf{S}}_{r k}^{T}[\mathbf{A}]^{T} \widetilde{\mathbf{u}}_{e k} d A-\sum_{k} \int_{A_{k}} \widetilde{\mathbf{S}}_{k}^{T} \widetilde{\Theta}_{0 k} d A=-\sum_{k} \int_{A_{k}} \widetilde{\mathbf{u}}_{e k}^{T}[\mathbf{A}] \widetilde{\mathbf{S}}_{r k} d A+ \\
& +\int_{L_{f}} \widetilde{\mathbf{u}}_{e}^{T}\left[A_{L}\right] \widetilde{\mathbf{S}}_{r} d L+\int_{L_{u}} \widetilde{\mathbf{u}}_{0}^{T}\left[A_{L}\right] \widetilde{\mathbf{S}}_{r} d L+\sum_{t} \int_{L_{t}} \widetilde{\mathbf{u}}_{e t}^{T}\left[A_{t}\right]\left(\widetilde{\mathbf{S}}_{r, \mathbf{u}}^{+}-\widetilde{\mathbf{S}}_{r, u}^{-}\right) d L-\sum_{k} \int_{A_{k}} \widetilde{\mathbf{S}}_{k}^{T} \widetilde{\Theta}_{0 k} d A
\end{aligned}
$$

преобразуется к виду

$$
\begin{aligned}
\Pi_{c} & =\sum_{k} \frac{1}{2} \int_{A_{k}} \widetilde{\mathbf{S}}_{r k}^{T}\left[d_{k}\right] \widetilde{\mathbf{S}}_{r k} d A+\sum_{k} \frac{1}{2} \int_{A_{k}} \widetilde{\mathbf{S}}_{e k}^{T}\left[d_{k}\right] \widetilde{\mathbf{S}}_{e k} d A+\sum_{k} \int_{A_{k}} \widetilde{\mathbf{S}}_{e k}^{T} \widetilde{\Theta}_{0 k} d A-\int_{L_{u}} \widetilde{\mathbf{u}}_{0}^{T}\left[A_{L}\right] \widetilde{\mathbf{S}}_{e} d L- \\
& -\sum_{k} \int_{A_{k}} \widetilde{\mathbf{u}}_{e k}^{T}[\mathbf{A}] \widetilde{\mathbf{S}}_{r k} d A+\sum_{t} \int_{L_{i}} \widetilde{\mathbf{u}}_{e t}^{T}\left[A_{t}\right]\left(\widetilde{\mathbf{S}}_{r, v t}^{+}-\widetilde{\mathbf{S}}_{r, v t}^{-}\right) d L+\int_{L_{f}} \widetilde{\mathbf{u}}_{e}^{T}\left[A_{L}\right] \widetilde{\mathbf{S}}_{r} d L .
\end{aligned}
$$

При заданных $\widetilde{\mathbf{S}}_{e k}$ значения второго, третьего и четвертого интегралов фиксированы, а три последних интеграла этого функционала для статически допустимых остаточных усилий равны нулю. Поэтому задача расчета остаточных усилий принимает такой вид:

$$
\sum_{k} \frac{1}{2} \int_{A_{k}} \widetilde{\mathbf{S}}_{r k}^{T}\left[d_{k}\right] \widetilde{\mathbf{S}}_{r k} d A \rightarrow \min
$$

при условиях

$$
\begin{array}{cccc}
\mathbf{f}\left(\widetilde{\mathbf{S}}_{e k}+\widetilde{\mathbf{S}}_{r k}\right) \leq \mathbf{f}_{0}\left(\widetilde{\mathbf{S}}_{0 k}\right) & \in A_{k}, \quad[\mathbf{A}] \widetilde{\mathbf{S}}_{r k}=\mathbf{0} & \in A_{k}, \\
\mathbf{\mathbf { f } _ { t }}\left(\widetilde{\mathbf{S}}_{e, v t}+\widetilde{\mathbf{S}}_{e, v t}\right) \leq \mathbf{f}_{0 t}\left(\widetilde{S}_{0 t}\right) & \in L_{t}, \quad\left[A_{L}\right] \widetilde{\mathbf{S}}_{r}=\mathbf{0} & \in L_{f}, \\
{\left[A_{t}\right] \widetilde{\mathbf{S}}_{r t}^{+}-\widetilde{\mathbf{S}}_{r, v t}=\mathbf{0} \quad \in L_{t},} & -\left[A_{t}\right] \widetilde{\mathbf{S}}_{r t}^{-}+\widetilde{\mathbf{S}}_{r, v t}=\mathbf{0} & \in L_{t} ; \\
k=1,2, \ldots, s ; & t=1,2, \ldots, z . &
\end{array}
$$


Функция цели этой эацачи выражает остаточную дополнительную энергию (дополнительную энергию остаточных усилий). В работе [1] она называется упругим потенциалом самонапряжений. Таким образом, задача (12) отвечает экстремальному принцигту [1]:

из всех статически допустимых векторов остаточных усилий действительным является тот вектор, при котором остапочная дополнительная энереия минимальна.

Очевидно, что задачи (8) и (12) тождественны. Первая из них Іолучается преобразованием второй и наоборот.

Методом множителей Лагранжа получается двойственная к (12) кинематическая формулировка задачи анализа остаточных параметров НДС:

$$
\begin{aligned}
& -\sum_{k} \frac{1}{2} \int_{A_{k}} \widetilde{\mathbf{S}}_{r k}^{T}\left[d_{k}\right] \widetilde{\mathbf{S}}_{r k} d A-\sum_{k} \int_{A_{k}} \tilde{\lambda}_{k}^{T}\left[f\left(\widetilde{\mathbf{S}}_{e k}+\widetilde{\mathbf{S}}_{r k}\right)\right] \widetilde{\mathbf{S}}_{r k} d A-\sum_{t} \int_{L_{t}} \tilde{\lambda}_{t}^{T}\left[f_{t}\left(\widetilde{\mathbf{S}}_{e, v t}+\widetilde{\mathbf{S}}_{r, v t}\right)\right] \widetilde{\mathbf{S}}_{r, v} d L+ \\
& +\sum_{k} \int_{A_{k}} \tilde{\lambda}_{k}^{T}\left\{\mathbf{f}\left(\widetilde{\mathbf{S}}_{e k}+\widetilde{\mathbf{S}}_{r k}\right)-\mathbf{f}_{0}\left(\widetilde{\mathbf{S}}_{0 k}\right)\right\} d A+\sum_{t} \int_{l_{t}} \tilde{\lambda}_{t}^{T}\left\{\mathbf{f}_{t}\left(\widetilde{\mathbf{S}}_{e, v}+\widetilde{\mathbf{S}}_{r, v}\right)-\mathbf{f}_{0 t}\left(\widetilde{\boldsymbol{S}}_{0 t}\right)\right\} d L \rightarrow \mathbf{m a x}
\end{aligned}
$$

при условиях

$$
\begin{aligned}
& {\left[d_{k}\right] \widetilde{\mathbf{S}}_{r k}+\left[f\left(\widetilde{\mathbf{S}}_{e k}+\widetilde{\mathbf{S}}_{r k}\right)\right]^{T} \widetilde{\lambda}_{k}-[\mathbf{A}]^{T} \widetilde{\mathbf{u}}_{r k}=\mathbf{0}, \quad \tilde{\lambda}_{k} \geq \mathbf{0} \quad \in A_{k}} \\
& {\left[f_{t}\left(\widetilde{\mathbf{S}}_{e, u}+\widetilde{\mathbf{S}}_{r, v t}\right)\right]^{T} \tilde{\lambda}_{t}-\widetilde{\mathbf{u}}_{n t}^{-}+\widetilde{\mathbf{u}}_{n t}^{+}=\mathbf{0}, \quad \tilde{\lambda}_{t} \geq \mathbf{0} \in I_{t} ;} \\
& {\left[A_{L}\right]^{T} \tilde{\mathbf{u}}_{r}=\mathbf{0} \quad \in L_{u} ; \quad k=1,2, \ldots, s ; \quad t=1,2, \ldots, z .}
\end{aligned}
$$

Неизвестными в ней являются функции остаточных усилий и перемещений, а также пластических множителей. Функщия цели, умноженная на -1 , означает полную энергию остаточных деформаций, а ее ограничения описывают область кинематически допустимых распределений остаточных перемещений. Поэтому математическая модель (13) соответствует экстремальному принцигу:

из всех кинематически допустимых распределений остаточных перемещениі действительным является по распределение, при котором полиая пергия оспаточных деформаций минимальна.

Математическую модель (13) также можно построить преобразованием задачи (9). Следовательно, минимальные принципы для остаточных усилий и перемешений тождественны минимальным принципам для полных усилий и персмещений, частными случаями которых являются хорошо известные в теории упругости вариационные принципы Кастильяна и Лагранжа. 13 случас линейных условий текучести можно построить более простые математические модели кинематической постановки задач, так как усилия можно исключить из числа неизвестных. 


\section{3. Дискретные равновесные математические модели}

Для численной реализации представленных задач необходимо построить их дискретные аналоги. Для этого могут применяться равновесные или геометрически совместные конечные элементы. Дискретные равновесные математические модели задач строятся на основании общих моделей статической постановки с применением равновесных конечных элементов (РКЭ). Далее метод РКЭ применительно к упругопластическим конструкциям разрабатывается и иллюстрируется на примере дискретизации задачи (6).

\section{1. Статические и геометрические уравнения равновесного элемегта}

Усилия в каждом элементе аптроксимируются вектор-функцией

$$
\mathbf{S}_{k}(\mathbf{x})=\sum_{k=1}^{\xi}\left[H_{k i}(\mathbf{x})\right] \mathbf{S}_{k i}=\left[H_{k}(\mathbf{x})\right] \mathbf{s}_{k}, \quad k=1,2, \ldots, s,
$$

где $\left[H_{k}(\mathbf{x})\right]$ - матрица функций формы (интерполяции) усилий $k$ - го элемегта; $\mathbf{S}_{k}-$ вектор усилий узловых точек элемента. Функция (14) должна удовлетворять требованиям сходимости [8]. Не ставится требование, чтобы она тождественно удовлетворяла дифференциальным условиям равновесия (1), так как эти условия могут быть удовлетворены в качестве дополнительных ограничений на дополнительную энергию элемента. Таким образом значительно упрощается и унифищируется построение интерполирующих функций усилий: появляется возможность применения классических методов интерполяции, которые широко применяются при построении совместных элементов. Выбор функций, тождественно удовлетворяющих уравнениям (1), довольно сложен и неуниверсален.

Вводится вектор обобщенных сил $\mathbf{P}_{k}$, работа которых на обобщенных перемещениях $\mathbf{u}_{k}$ должна быть эквивалентна работе внутренних сил. Обобценные силы не независимы, так как должны удовлетворять уравнениям равновесия элемента

$$
\mathbf{P}_{k}=\left[A_{k}\right] \mathbf{Q}_{k} \quad \text { или } \quad \mathbf{P}_{k}=\left[A_{s k}\right] \mathbf{S}_{k}+\left[A_{v k}\right] \mathbf{S}_{v k},
$$

где $\mathbf{Q}_{k} \equiv\left\{\mathbf{S}_{k}, \mathbf{S}_{v k}\right\}^{T}$ - расширенный вектор узловых усилий, в который включены поверхностные усилия элемента, необходимые для построения условий (2). Уравнение (15) определяет связь между обобщенными силами $\mathbf{P}_{k}$ и узловыми усилиями элеменга. Оно включает в себя уравнения равновесия внугри, если 
функция (14) тождественно не удовлетворяет условиям (1), и на границах элемента. Обобщенные силы и перемещения выбираются с таким расчетом, чтобы уравнения равновесия дискретной модели конструкции, составленные по направлению этих перемещений, гарантировали удовлетворение условий равновесия во всех точках внутри и на границах элементов.

Геометрические уравнения элемента строятся на основе принципа виртуальных сил, согласно которому

$$
\begin{aligned}
\left\{\partial \mathbf{P}_{k}\right\}^{T} \mathbf{u}_{k} & =\int_{A_{k}}\left\{\partial \mathbf{S}_{k}(\mathbf{x})\right\}^{T}\left\{\left[d_{k}\right] \mathbf{S}_{k}(\mathbf{x})+\Theta_{0 k}(\mathbf{x})+\left[f\left(\mathbf{S}_{k}(\mathbf{x})\right)\right]^{T} \lambda_{k}(\mathbf{x})\right\} d A+ \\
& +\int_{L_{l d}}\left\{\partial \mathbf{S}_{v k}(\mathbf{x})\right\}^{T}\left[f_{t}\left(\mathbf{S}_{v k}(\mathbf{x})\right)\right]^{T} \lambda_{k t}(\mathbf{x}) d L .
\end{aligned}
$$

Учитывая зависимости (14), (15) и произвольность вариаций $\partial \mathbf{S}_{k}, \partial \mathbf{S}_{\mathfrak{k}}$, они получают такой общий вид:

$$
\begin{aligned}
& {\left[D_{k}\right] \mathbf{s}_{k}+\Theta_{0 k}+\Theta_{p k}-\left[A_{s k}\right]^{T} \mathbf{u}_{k}=\mathbf{0},} \\
& \Theta_{p \downarrow k}-\left[A_{\imath k}\right]^{T} \mathbf{u}_{k}=\mathbf{0}
\end{aligned}
$$

Здесь матрица податливости элемента

$$
\left[D_{k}\right]=\iint_{A_{k}}\left[H_{k}(\mathbf{x})\right]^{T}\left[d_{k}\right]\left[H_{k}(\mathbf{x})\right] d A,
$$

векторы взвешенных узловых начальных и пластических деформаций

$$
\begin{gathered}
\Theta_{0 k}=\int_{A_{k}}\left[H_{k}(\mathbf{x})\right]^{T} \Theta_{0 k}(\mathbf{x}) d A, \\
\Theta_{p k}=\sum_{i=1}^{\xi}\left[\widetilde{\Phi}_{k i}\right] \lambda_{k i}, \quad \Theta_{p v k}=\sum_{t}\left[\widetilde{\Phi}_{v k t}\right] \lambda_{k t}, \\
{\left[\widetilde{\Phi}_{k i}\right]=\iint_{A_{k}}\left[H_{k}(\mathbf{x})\right]^{T}\left[f\left(\mathbf{s}_{k}(\mathbf{x})\right)\right]^{T}\left[H_{\lambda k i}(\mathbf{x})\right] d A,} \\
\lambda_{k}(\mathbf{x})=\sum_{i=1}^{\xi}\left[H_{\lambda k i}(\mathbf{x})\right] \lambda_{k i}=\left[H_{\lambda k}(\mathbf{x})\right] \lambda_{k}, \\
{\left[\widetilde{\Phi}_{v k t}\right]=\int\left[H_{\nu k}(\mathbf{x})\right]^{T}\left[f_{t}\left(\mathbf{S}_{\imath k}(\mathbf{x})\right)\right]^{T}\left[H_{\lambda k t}(\mathbf{x})\right] d L,}
\end{gathered}
$$

$\lambda_{k i} \geq \mathbf{0}, \quad \lambda_{k t} \geq \mathbf{0} ; \xi$ - число узлов элемента.

При заданных силах $\mathbf{P}_{k}$, начальных и пластических деформациях система уравнений (15), (16) однозначно определяет НдС элемента. 


\section{2. Условия текучести элемента}

Условия текучести (4) должны соблюдаться во всех внутренних точках элемента, а условия (5) - во всех его граничных точках. Однако при расчете континуальных конструкций это требование выполнить невозможно. Поэтому условия текучести удовлетворяются в слабой форме [9]. Часто используемые точечные условия текучести являются одним примером слабой формы условий текучести.

Слабая форма условий текучести может быть построена классическими методами коллокаций. Она получает такой общий вид:

$$
\int_{A_{k}}\left[G_{k}(\mathbf{x})\right]^{T}\left\{\mathbf{f}_{0}\left(S_{0 k}(\mathbf{x})\right)-\mathbf{f}\left(\mathbf{S}_{k}(\mathbf{x})\right)\right\} d A \geq \mathbf{0} \quad \in A_{k},
$$

где $\left[G_{k}(\mathbf{x})\right]$ - матрица весовых функций, подматрицами которой являются матрицы $\left[G_{k i}(\mathbf{x})\right]$, соответствующие узлам $i=1,2, \ldots, \xi$. На основе (22) и (14) получается следующее дискретное выражение условий текучести элемента:

где

$$
\begin{gathered}
\mathbf{f}_{0 k}\left(\mathbf{S}_{0 k}\right)-\mathbf{f}_{k}\left(\mathbf{S}_{k}\right) \geq \mathbf{0}, \\
\mathbf{f}_{k}\left(\mathbf{S}_{k}\right)=\int\left[G_{A_{k}}(\mathbf{x})\right]^{T} \mathbf{f}\left(\mathbf{S}_{k}(\mathbf{x})\right) d A, \\
\mathbf{f}_{0 k}\left(\mathbf{S}_{0 k}\right)=\int_{A_{k}}\left[G_{k}(\mathbf{x})\right]^{T} \mathbf{f}_{0}\left(S_{0 k}(\mathbf{x})\right) d A, \\
S_{0 k}(\mathbf{x})=\mathbf{H}_{0 k}^{T}(\mathbf{x}) \mathbf{S}_{0 k} .
\end{gathered}
$$

Выбор разных весовых функщий приводит к разным выражениям векторов $\mathbf{f}_{k}\left(\mathbf{S}_{k}\right)$, $\mathbf{f}_{0}\left(\mathbf{S}_{0 k}\right)$ и разным условиям текучести элемента. Весовые функции могут быть выбраны в соответствии с классическими методами коллокаций [10].

Согласно методу коллокации в точке пологается $\left[G_{k i}\left(\mathbf{x}_{i}\right)\right]=[E]$ для узловой точки $i$ и $\left[G_{k i}\left(\mathbf{x}_{j}\right)\right]=[0]$ во всех остальных точках элемента. Тогда условия текучести элемента выражаются условиями текучести всех его узловых точек:

$$
\mathbf{f}_{0 k i}\left(S_{0 k i}\right)-\mathbf{f}_{k i}\left(\mathbf{S}_{k i}\right) \geq \mathbf{0}, \quad i=1,2, \ldots, \xi
$$


Составляющими вектора $\mathbf{f}_{k}\left(\mathbf{S}_{k}\right)$ являются функщии $\mathbf{f}_{k i}\left(\mathbf{S}_{k i}\right), \quad i=1,2, \ldots, \xi$. Используя метод коллокации в подобласти, принимаются постоянные единичные весовые функции $\left[G_{k}(\mathbf{x})\right]=[E]$ во всей области элемента. Тогда

где

$$
\begin{aligned}
& \mathbf{f}_{k}\left(\mathbf{S}_{k}\right)=\int_{A_{k}} \mathbf{f}\left(\mathbf{S}_{k}(\mathbf{x})\right) d A \leq \mathbf{f}_{0 k}\left(\mathbf{S}_{0 k}\right), \\
& \mathbf{f}_{0 k}\left(\mathbf{S}_{0 k}\right)=\int_{A_{k}} \mathbf{f}_{0}\left(S_{0 k}(\mathbf{x})\right) d A
\end{aligned}
$$

В работах $[11,12]$ такое условие текучести независимо было построено для критерия прочности Хубера-Мизеса, исходя из энергетических соображений.

Качественно новое условие текучести конечного элемента получается методом коллокации Бубнова-Галеркина [9]. Для каждого узла элемента выбираются матрицы весовых функций

$$
\left[G_{k i}(\mathbf{x})\right]=\left[\operatorname{diag} H_{j k i}(\mathbf{x})\right]
$$

где $H_{j k i}(\mathbf{x})$ - функция формы усилия $i$-го узла, которое входит в услювие текучести $j$ и аппроксимируется полиномом наивысшей степени. Тогда условия текучести всего элемента выражаются интегральными условиями текучести его узловых точек:

где

$$
\mathbf{f}_{k i}\left(\mathbf{S}_{k}\right)=\int_{A_{k}}\left[G_{k i}(\mathbf{x})\right] \mathbf{f}\left(\mathbf{S}_{k}(\mathbf{x})\right) d A \leq \mathbf{f}_{0 k i}\left(\mathbf{S}_{0 k}\right), \quad i=1,2, \ldots, \xi
$$

При этом составляющими векторов $\mathbf{f}_{k}\left(\mathbf{S}_{k}\right), \mathbf{f}_{0 k}\left(\mathbf{S}_{0 k}\right)$ являются векторы $\mathbf{f}_{k i}\left(\mathbf{S}_{k}\right)$, $\mathbf{f}_{0 k i}\left(\mathbf{S}_{0 k}\right), i=1,2, \ldots, \xi$. Если функция текучести скалярная, то матрица $\left[G_{k i}(\mathbf{x})\right]$ превращается в скаляр $G_{k i}(\mathbf{x})$. Задавая аппроксимирующие функции

$$
\mathbf{f}\left(\mathbf{S}_{k}(\mathbf{x})\right)=\sum_{i=1}^{\xi}\left[H_{f k i}(\mathbf{x})\right] \mathbf{f}_{k i}\left(\mathbf{S}_{k i}\right)=\left[H_{f k}(\mathbf{x})\right] \mathbf{f}_{k}\left(\mathbf{S}_{k}\right)
$$

можно построить упрощенные выражения интегральных условий текучести [9].

Рассмотренные методы дискретизации условия текучести элемента (4) могут применяться и для дискретизации условий текучести граней элемента (5). Дискретные выражения условия текучести грани $t$ элемента выражаются неравенством

$$
\mathbf{f}_{t}\left(\mathbf{S}_{v t}^{+}\right) \leq \mathbf{f}_{0 t}\left(\mathbf{S}_{0 t}^{+}\right) \quad \text { или } \quad \mathbf{f}_{t}\left(\mathbf{S}_{v t}^{-}\right) \leq \mathbf{f}_{0 t}\left(\mathbf{S}_{0 t}^{-}\right)
$$


где

$$
\begin{array}{ll}
\mathbf{f}_{t}\left(\mathbf{S}_{v^{t}}^{+}\right)=\int_{L_{t}}\left[G_{t}(\mathbf{x})\right]^{T} \mathbf{f}\left(\mathbf{S}_{v t}^{+}(\mathbf{x})\right) d L, & \mathbf{f}_{0 t}\left(\mathbf{S}_{0 t}^{+}\right)=\int_{L_{t}}\left[G_{t}(\mathbf{x})\right]^{T} \mathbf{f}_{0 t}\left(\mathbf{s}_{0 t}^{+}(\mathbf{x})\right) d L, \\
\mathbf{f}_{t}\left(\mathbf{S}_{v t}^{-}\right)=\int_{L_{t}}\left[G_{t}(\mathbf{x})\right]^{T} \mathbf{f}\left(\mathbf{S}_{v t}^{-}(\mathbf{x})\right) d L, & \mathbf{f}_{0 t}\left(\mathbf{S}_{0 t}^{-}\right)=\int_{L_{t}}\left[G_{t}(\mathbf{x})\right]^{T} \mathbf{f}_{0 t}\left(\mathbf{S}_{0 t}^{-}(\mathbf{x})\right) d L .
\end{array}
$$

Выбирая весовые функции согласно разным методам коллокаций, получим разные выражения дискретных условий текучести (33) для граней конечных элементов, аналогичные условиям (27), (28) и (30).

\section{3. Анализ дискретньх условий текучести}

Рассмотрены три способа дискретизации условий текучести. Чаще всего в настоящее время используемые точечные условия текучести соответствуют методу точечной коллокации. Они обусловливают удовлетворение условий (4), (5) только в узлах конечных элементов. Интегральные условия текучести не гарантируют соблюдения условий (4), (5) в узловых точках. Однако известно, что дискретизация дифференциальных или функциональных зависимостей методом коллокации Бубнова-Галеркина приводит $\mathbf{k}$ наименышей суммарной погрешности. Ниже это подтверждается качественным исследованием дискретных условий текучести, построенных разными способами для критерия прочностн Хубера-Мизеса

$$
\mathbf{f}_{k}(\mathbf{x})=\mathbf{S}_{k}^{T}(\mathbf{x})[\Pi] \mathbf{S}_{k}(\mathbf{x}) \leq S_{0 k}^{2}(\mathbf{x})
$$

Точное выражение вектора пластических деформаций, отвечающего этим условиям текучести,

$$
\Theta_{p k}=\sum_{i=1}^{\xi} 2 \lambda_{k i} \int_{A_{k}} H_{\lambda k i}(\mathbf{x})\left[H_{k}(\mathbf{x})\right]^{T}[\Pi]\left[H_{k}(\mathbf{x})\right] \mathbf{S}_{k} d A=\sum_{i=1}^{\xi} 2 \lambda_{k i}\left[\Phi_{k i}\right] \mathbf{S}_{k}
$$

где

$$
\left[\Phi_{k l}\right]=\int_{A_{k}} H_{\lambda k i}(\mathbf{x})\left[H_{k}(\mathbf{x})\right]^{T}[\Pi]\left[H_{k}(\mathbf{x})\right] d A .
$$

Оценка условий текучести осуществляется сравнением выражения (37) с выражениями векторов пластических деформаций, ассоциированных с дискретными условиями текучести Хубера-Мизеса.

Согласно вышеприведенным зависимостям получаются следующие выражения дискретных условий текучести и отвечающцх им векторов пластических деформаций:

а) точечная коллокация -

$$
\mathbf{f}_{k i}\left(\mathbf{S}_{k i}\right)=\mathbf{S}_{k i}^{T}[\Pi] \mathbf{S}_{k i} \leq S_{0 k i}^{2}, \quad i=1,2, \ldots, \xi
$$




$$
\Theta_{p k}=\left[\operatorname{diag} 2 \lambda_{k i}[\Pi]\right] \mathbf{s}_{k}=\left[\Pi_{k}\right] \mathbf{s}_{k}
$$

б) коллокация в подобласти -

$$
\begin{gathered}
\mathbf{f}_{k}\left(\mathbf{s}_{k}\right)=\mathbf{S}_{k}^{T}\left[\Phi_{k}\right] \mathbf{s}_{k} \leq \mathbf{S}_{0 k}^{T}\left[\Gamma_{k}\right] \mathbf{S}_{0 k}, \\
\Theta_{p k}=2 \lambda_{k}\left[\Phi_{k}\right] \mathbf{s}_{k}, \\
\text { где }\left[\Phi_{k}\right]=\int_{A_{k}}\left[H_{k}(\mathbf{x})\right]^{T}[\Pi]\left[H_{k}(\mathbf{x})\right] d A, \quad\left[\Gamma_{k}\right]=\int_{A_{k}} \mathbf{H}_{0 k}(\mathbf{x}) \mathbf{H}_{0 k}^{T}(\mathbf{x}) d A ;
\end{gathered}
$$

в) коллокация Бубнова-Галеркина -

$$
\begin{gathered}
\mathbf{f}_{k i}\left(\mathbf{S}_{k}\right)=\mathbf{S}_{k}^{T}\left[\Phi_{k i}\right] \mathbf{S}_{k} \leq \mathbf{S}_{0 k}^{T}\left[\Gamma_{k i}\right] \mathbf{S}_{0 k}, \quad i=1,2, \ldots, \xi ; \\
\Theta_{p k}=\sum_{i=1}^{\xi} 2 \lambda_{k i}\left[\Phi_{k i}\right] \mathbf{S}_{k},
\end{gathered}
$$

где

$$
\left[\Phi_{k i}\right]=\int_{A_{k}} G_{k i}(\mathbf{x})\left[H_{k}(\mathbf{x})\right]^{T}[\Pi]\left[H_{k}(\mathbf{x})\right] d A, \quad\left[\Gamma_{k i}\right]=\int_{A_{k}} G_{k i}(\mathbf{x}) \mathbf{H}_{0 k}(\mathbf{x}) \mathbf{H}_{0 k}^{T}(\mathbf{x}) d A
$$

Выражения (39) и (40) отличаются от точного выражения вектора пластических деформаций (37). Разница между ними является погрешностыю дискретизации условий текучести методами точечной коллокации и коллокации в подобласти. Еслю принять $H_{\lambda k i}(\mathbf{x})=G_{k i}(\mathbf{x})$, то выражения (37) и (41) полностью совпадают, т. е. выражение вектора $\Theta_{p k}$, определенное для дискретных условий текучести, совпадает с выражением этого вектора, определенного для функциональных условий текучести (36). Эти выводы действительны не только для условий (36), но и для любых условий текучести. Это свидетельствует о том, что метод коллокации Бубнова-Галеркина дает наиболее точную аппроксимацию условий текучести и пластических деформаций, что позволяет наиболее точно определить деформированное состояние конструкции. Менее точны точечные условия текучести (27). Однако они отличаются простотой. Наименышая точность аппроксимации метода коллокации в подобласти.

Выясним физический смысл пластических множителей, отвечающих дискретным условиям текучести. В случае точечных условий текучести

$$
\int_{A_{k}} \lambda_{k}^{T}(\mathbf{x}) \mathbf{f}\left(\mathbf{S}_{k}(\mathbf{x})\right) d A \approx \sum_{i=1}^{\xi} \int_{A_{k}} \lambda_{k}^{T}(\mathbf{x})\left[H_{f k i}(\mathbf{x})\right] \mathbf{f}_{k i}\left(\mathbf{S}_{k i}\right) d A=\sum_{i=1}^{\xi} \lambda_{k i}^{T} \mathbf{f}_{k i}\left(\mathbf{S}_{k i}\right) .
$$

Отсюда

$$
\lambda_{k i}=\iint_{A_{k}}\left[H_{f k i}(\mathbf{x})\right]^{T} \lambda_{k}(\mathbf{x}) d A .
$$


Здесь имеют место не локальные, а взвешенные пластические множители. Следовательно, вектор $\Theta_{p k}$ выражает не действительные пластические деформации в узлах элемента, а лишь их взвешенные значения, представляющие собой суммарные деформации окрестностей узловых точек элемента. Несмотря на это, после решения задачи можно определить и действительные значения пластических множителей в узлах элемента, решая систему уравнений

$$
\int\left[H_{A_{k}}\left[H_{f k i}(\mathbf{x})\right]^{T}\left[H_{\lambda k}(\mathbf{x})\right] \lambda_{k} d A=\lambda_{k i}^{*}, \quad i=1,2, \ldots, \xi\right.
$$

где $\lambda_{k i}^{*}$ - вектор вэвешенных пластических множителей, полученный решением задачи. После этого могуг быть определены и действительные значения пластических деформаций.

Для интегральных условий текучести элемента в целом

$$
\int_{A_{k}} \lambda_{k}^{T}(\mathbf{x}) \mathbf{f}\left(\mathbf{S}_{k}(\mathbf{x})\right) d A \approx \lambda_{k}^{T} \int_{A_{k}} \mathbf{f}\left(\mathbf{S}_{k}(\mathbf{x})\right) d A=\lambda_{k}^{T} \mathbf{f}_{k}\left(\mathbf{S}_{k}\right)
$$

Отсюда $\lambda_{k}(\mathbf{x})=\lambda_{k}=$ const $\in A_{k} . \quad$ Для интегральных узловых условий текучести

$$
\int_{A_{k}} \lambda_{k}^{T}(\mathbf{x}) \mathbf{f}\left(\mathbf{S}_{k}(\mathbf{x})\right) d A \approx \sum_{i=1}^{\xi} \lambda_{k i}^{T} \int_{A_{k}}\left[H_{\lambda k i}(\mathbf{x})\right] \mathbf{f}\left(\mathbf{S}_{k}(\mathbf{x})\right) d A=\sum_{i=1}^{\xi} \lambda_{k i}^{T} \int_{A_{k}}\left[G_{k i}(\mathbf{x})\right] \mathbf{f}\left(\mathbf{S}_{k}(\mathbf{x})\right) d A=\sum_{i=1}^{\xi} \lambda_{k i}^{T} \mathbf{f}_{k i}\left(\mathbf{S}_{k}\right),
$$

тогда

$$
\left[G_{k i}(\mathbf{x})\right]=\left[H_{\lambda k i}(\mathbf{x})\right]
$$

Вышесказанное позволяет сделать следующие выводы. Дискретизация условий текучести методом точечной коллокации соответствует предположению о принятии аппроксимаций (32). Применение метода коллокации в подобласти соответствует предположению о постоянстве пластических множителей во всей подобласти. Построение же условий текучести методом Бубнова-Галеркина означает принятие функций формы пластических множителей или усилий в качестве весовых функций. Это еще раз подтверждает вывод о том, что с точки зрения теоретической и точности оценки НДС предпочтительны интегральные узловые условия текучести, хотя в практическом отношгении наиболее просты точечные условия текучести. Для обоих видов интегральных условий текучести происхождение пшастических множителей локальное. Однако в геометрических уравнениях все равно присутствуют взвешенные пластические, как и другие, деформации. 


\section{4. Математические модели}

Прямая постановка. Внешние воздействия дискретной модели конструкции описываются векторами $\mathbf{F}, \Theta_{0}$ и $\mathbf{u}_{0}$, а перемещения - вектором u. НДС всех конечных элементов описывается векторами

$$
\begin{aligned}
& \mathbf{P} \equiv\left\{\mathbf{P}_{1}, \mathbf{P}_{2}, \ldots, \mathbf{P}_{s}\right\}^{T}, \quad \mathbf{S} \equiv\left\{\mathbf{s}_{1}, \mathbf{s}_{2}, \ldots, \mathbf{s}_{s}\right\}^{T}, \quad \mathbf{s}_{v} \equiv\left\{\mathbf{s}_{v 1}^{+}, \mathbf{s}_{v 1}^{-}, \mathbf{S}_{v 2}^{+}, \mathbf{s}_{v 2}^{-}, \ldots, \mathbf{s}_{v z}^{+}, \mathbf{s}_{v z}^{-}\right\}^{T}, \\
& \overline{\mathbf{u}} \equiv\left\{\mathbf{u}_{1}, \mathbf{u}_{2}, \ldots, \mathbf{u}_{s}\right\}^{T}, \quad \lambda \equiv\left\{\lambda_{1}, \lambda_{2}, \ldots, \lambda_{s}\right\}^{T}, \quad \lambda_{v} \equiv\left\{\lambda_{1}^{+}, \lambda_{1}^{-}, \lambda_{2}^{+}, \lambda_{2}^{-}, \ldots, \lambda_{z}^{+}, \lambda_{z}^{-}\right\}^{T}, \\
& \mathbf{S}_{0} \equiv\left\{\mathbf{s}_{01}, \mathbf{s}_{02}, \ldots, \mathbf{S}_{0 s}\right\}^{T}, \quad \Theta_{0} \equiv\left\{\Theta_{01}, \Theta_{02}, \ldots, \Theta_{0 s}\right\}^{T}
\end{aligned}
$$

и зависимостями

$$
\begin{aligned}
& \mathbf{P}=\left[\bar{A}_{s}\right] \mathbf{S}+\left[\bar{A}_{v}\right] \mathbf{S}_{v}, \quad \mathbf{f}(\mathbf{S}) \leq \mathbf{f}_{0}\left(\mathbf{S}_{0}\right), \quad \mathbf{f}_{v}\left(\mathbf{S}_{v}\right) \leq \mathbf{f}_{0 v}\left(\mathbf{S}_{0}\right), \\
& {[D] \mathbf{S}+\Theta_{0}+[f(\mathbf{S})]^{T} \lambda-\left[\bar{A}_{s}\right]^{T} \overline{\mathbf{u}}=\mathbf{0}, \quad\left[f_{v}\left(\mathbf{S}_{v}\right)\right]^{T} \lambda_{v}-\left[\bar{A}_{v}\right]^{T} \overline{\mathbf{u}}=\mathbf{0},}
\end{aligned}
$$

где $[f(\mathbf{S})],\left[f_{v}\left(\mathbf{S}_{v}\right)\right]$ - матрицы градиентов условий текучести конечных элементов и их граней; $\left[\bar{A}_{s}\right],\left[\bar{A}_{v}\right],[D]$ - квазидиагональные матрицы, диагональными блоками которых являются матрицы $\left[A_{s k}\right],\left[A_{v k}\right],\left[D_{k}\right]$. Здесь уравнения одного элемента не связаны с соответствующими уравнениями других элементов.

Объединение свободных конечных элементов в дискретную модель конструкции осуществляется при помощи уравнений совместности перемещений

$$
\overline{\mathbf{u}}=[C] \mathbf{u}+\left[C_{0}\right] \mathbf{u}_{0} .
$$

Этими уравнениями определяется связь между перемещениями узлов элементов и дискретной модели. Уравнения равновесия дискретной модели строятся на основе принципа возможных перемещений, согласно которому

$$
\{\partial \overline{\mathbf{u}}\}^{T} \mathbf{P}=\{\partial \mathbf{u}\}^{T} \mathbf{F} .
$$

Учитывая зависимости (42), (43) и произвольность вариации ди, они получают такой вид

$$
\begin{gathered}
{[C]^{T} \mathbf{P}=\mathbf{F}} \\
{\left[A_{s}\right] \mathbf{S}+\left[A_{v}\right] \mathbf{S}_{\mathbf{v}}=\mathbf{F},} \\
\text { или } \\
\text { где }\left[A_{s}\right]=[C]^{T}\left[\bar{A}_{s}\right], \quad\left[A_{v}\right]=[C]^{T}\left[\bar{A}_{v}\right] .
\end{gathered}
$$

Для узлов на опорном контуре, где заданы перемещения $\mathbf{u}_{0}$, составляются уравнения равновесия 


$$
\left[A_{0 s}\right] \mathbf{S}+\left[A_{0 v}\right] \mathbf{S}_{v}=\mathbf{F}_{r},
$$

где $\mathbf{F}_{r}$ - вектор опорных реакций;

$$
\left[A_{0 s}\right]=\left[C_{0}\right]^{T}\left[\bar{A}_{s}\right], \quad\left[A_{0 v}\right]=\left[C_{0}\right]^{T}\left[\bar{A}_{v}\right]
$$

Матрицы $[C]$ и $\left[C_{0}\right]$ булевые. Поэтому уравнения равновесия (45) и (47) могуг быть построены прямым формированием матриц $\left[A_{s}\right],\left[A_{v}\right] \cdot\left[A_{0 s}\right]$ и $\left[A_{0 v}\right]$.

Используя зависимости (42)-(48), цля дискретной модели конструкции получаются следующие уравнения геометрической совместности

$$
\begin{aligned}
& {[D] \mathbf{S}+[f(\mathbf{S})]^{T} \lambda-\left[A_{S}\right]^{T} \mathbf{u}=\left[A_{0 s}\right]^{T} \mathbf{u}_{0}-\boldsymbol{\Theta}_{0},} \\
& {\left[f_{v}\left(\mathbf{S}_{v}\right)\right]^{T} \lambda_{v}-\left[A_{v}\right]^{T} \mathbf{u}=\mathbf{0} .}
\end{aligned}
$$

Тогда задача анализа НДС упругопластической конструкции при однократном нагружении может быть представлена системой уравнений

$$
\begin{aligned}
& {\left[A_{s}\right] \mathbf{S}+\left[A_{v}\right] \mathbf{S}_{v}=\mathbf{F}, \quad[D] \mathbf{S}+[f(\mathbf{S})]^{T} \lambda-\left[A_{s}\right]^{T} \mathbf{u}=\left[A_{0 s}\right]^{T} \mathbf{u}_{0}-\Theta_{0},} \\
& {\left[f_{v}\left(\mathbf{S}_{v}\right)\right]^{T} \lambda_{v}-\left[A_{v}\right]^{T} \mathbf{u}=\mathbf{0}, \quad \mathbf{f}(\mathbf{S}) \leq \mathbf{f}_{0}\left(\mathbf{S}_{0}\right), \quad \mathbf{f}_{v}\left(\mathbf{S}_{v}\right) \leq \mathbf{f}_{0 v}\left(\mathbf{S}_{0}\right),} \\
& \lambda^{T}\left\{\mathbf{f}_{0}\left(\mathbf{S}_{0}\right)-\mathbf{f}(\mathbf{S})\right\}=0, \quad \lambda_{v}^{T}\left\{\mathbf{f}_{0 v}\left(\mathbf{S}_{0}\right)-\mathbf{f}_{v}\left(\mathbf{S}_{v}\right)\right\}=0, \quad \lambda \geq \mathbf{0}, \quad \lambda_{v} \geq \mathbf{0} .
\end{aligned}
$$

Неизвестными в ней являются векторы усилий $\mathbf{S}$ и $\mathbf{S}_{v}$, вектор перемещений $\mathbf{u}$ и векторы пластических множителей $\lambda, \lambda_{v}$. Математическая модель получена прямым построением уравнений. Поэтому ее можно назвать прямой постановкой задачи анализа НдС.

При заданных пластических деформациях $\Theta_{p}, \Theta_{p v}$ НДС конструкции описывается системой уравнений

$$
\begin{aligned}
& {\left[A_{s}\right] \mathbf{S}+\left[A_{v}\right] \mathbf{S}_{v}=\mathbf{F}, \quad\left[A_{v}\right]^{T} \mathbf{u}=\Theta_{p v},} \\
& {[D] \mathbf{S}-\left[A_{s}\right]^{T} \mathbf{u}=\left[A_{0 s}\right]^{T} \mathbf{u}_{0}-\Theta_{0}-\Theta_{p} .}
\end{aligned}
$$

Решением этой задачи являются вектор перемешений

$$
\mathbf{u}=\left\{[K]^{-1}-[K]^{-1}\left[A_{v}\right]\left[K_{v}\right]^{-1}\left[A_{v}\right]^{T}[K]^{-1}\right\} \mathbf{F}_{\mathbb{\Sigma}}+[K]^{-1}\left[A_{v}\right]\left[K_{v}\right]^{-1} \Theta{ }_{p v}=[\beta] \mathbf{F}_{\mathbb{I}}+[K]^{-1} \mathbf{F}_{v}
$$


или

$$
\mathbf{u}=[\beta] \mathbf{F}+[H]\left\{\Theta_{p}+\Theta_{0}-\left[A_{0 s}\right]^{T} \mathbf{u}_{0}\right\}+\left[H_{v}\right] \Theta_{p v}
$$

и векторы усилий

$$
\begin{gathered}
\mathbf{S}_{v}=\left[K_{v}\right]^{-1}\left[A_{v}\right]^{T}[K]^{-1} \mathbf{F}_{\Sigma}-\left[K_{v}\right]^{-1} \Theta_{p v}=\left[H_{v}\right]^{T} \mathbf{F}_{\Sigma}-\left[K_{v}\right]^{-1} \Theta_{p v} \\
\mathbf{S}=[D]^{-1}\left\{\left[A_{S}\right]^{T} \mathbf{u}+\left[A_{0 s}\right]^{T} \mathbf{u}_{0}\right\}-[D]^{-1} \Theta_{p}
\end{gathered}
$$

или

$$
\begin{gathered}
\mathbf{S}_{v}=\left[H_{v}\right]^{T} \mathbf{F}+\left[H_{v}\right]^{T}\left[A_{s}\right][D]^{-1}\left\{\Theta_{p}+\Theta_{0}-\left[A_{0 s}\right]^{T} \mathbf{u}_{0}\right\}-\left[K_{v}\right]^{-1} \Theta_{p v}, \\
\mathbf{S}=[\alpha] \mathbf{F}+[G]\left\{\Theta_{p}+\Theta_{0}-\left[A_{0 s}\right]^{T} \mathbf{u}_{0}\right\}+\left[G_{v}\right] \Theta_{p v}
\end{gathered}
$$

Здесь

$$
\begin{aligned}
& {[K]=\left[A_{s}\right][D]^{-1}\left[A_{s}\right]^{T}, \quad\left[K_{v}\right]=\left[A_{v}\right]^{T}[K]^{-1}\left[A_{v}\right], \quad[\alpha]=[D]^{-1}\left[A_{s}\right]^{T}[\beta],} \\
& {[\beta]=[K]^{-1}-[K]^{-1}\left[A_{v}\right]\left[K_{v}\right]^{-1}\left[A_{v}\right]^{T}[K]^{-1}, \quad \mathbf{F}_{v}=\left[A_{v}\right]\left[K_{v}\right]^{-1} \Theta_{p v},} \\
& \mathbf{F}_{\Sigma}=\mathbf{F}+\mathbf{F}_{0}+\mathbf{F}_{p}-\mathbf{F}_{u}=\mathbf{F}+\left[A_{s}\right][D]^{-1} \Theta_{0}+\left[A_{s}\right][D]^{-1} \Theta_{p}-\left[A_{s}\right][D]^{-1}\left[A_{0 s}\right]^{T} \mathbf{u}_{0}, \\
& {[H]=[\beta]\left[A_{s}\right][D]^{-1}=[\alpha]^{T}, \quad[G]=[D]^{-1}\left[A_{s}\right]^{T}[\beta]\left[A_{s}\right][D]^{-1}-[D]^{-1},} \\
& {\left[H_{v}\right]=[K]^{-1}\left[A_{v}\right]\left[K_{v}\right]^{-1} ; \quad\left[G_{v}\right]=[D]^{-1}\left[A_{s}\right]^{T}[K]^{-1}\left[A_{v}\right]\left[K_{v}\right]^{-1} .}
\end{aligned}
$$

Остаточные усилия и перемещения описываются следующей системой уравнений

$$
\begin{aligned}
& {\left[A_{s}\right] \mathbf{S}_{r}+\left[A_{v}\right] \mathbf{S}_{r v}=\mathbf{0},} \\
& {[D] \mathbf{S}_{r}-\left[A_{s}\right]^{T} \mathbf{u}_{r}=-\Theta_{p}, \quad\left[A_{v}\right]^{T} \mathbf{u}_{r}=\Theta_{p v} .}
\end{aligned}
$$

Решая эту систему, получаем

$$
\begin{gathered}
\mathbf{u}_{r}=[H] \Theta_{p}+\left[H_{v}\right] \Theta_{p v}, \\
\mathbf{S}_{r}=[G] \Theta_{p}+\left[G_{v}\right] \Theta_{p v}, \\
\mathbf{S}_{r v}=\left[H_{v}\right]^{T}\left[A_{s}\right][D]^{-1} \Theta_{p}-\left[K_{v}\right]^{-1} \Theta_{p v}
\end{gathered}
$$

Остаточные перемещения и усилия здесь однозначно выражаются через пластические деформации. В этих зависимостях учитываются пластические деформации в поверхностях раздела конечных элементов. Этим выражения (60) и (61) отличаются от представленных в [3] выражений остаточных усилий и перемещений, где учитываются лишь пластические деформации внутри элементов. 
Зависимости (51)-(56) и (60)-(62) позволяют определить усилия и перемецения конструкцции от разных внешпих воздействий, в качестве которых могут быть и пластические деформации. Однако они могуг быть применены лишь в тех случаях, когда пластические деформации известны или могут быть определены решением отдельной задачи как, например, в случае линейных условий текучести (см. ниже). Тогда их применение целесообразно.

Вариационные постановки. Решение прямой постановки задачи (49) затруднено из-за болышого числа неизвестных и уравнений, а также присутствия неравенств. На основе вариационного принципа о минимуме полной дополнительной энергии и метода множителей Лагранжа строится двойственная пара дискретных равновесных математических моделей задачи определения суммарных усилий и перемещений.

Статическая постановка задачи -

$$
\frac{1}{2} \mathbf{S}^{T}[D] \mathbf{S}+\mathbf{S}^{T} \Theta_{0}-\mathbf{S}^{T}\left[A_{0 s}\right]^{T} \mathbf{u}_{0} \rightarrow \mathbf{m i n}
$$

при условиях

$$
\mathbf{f}_{0}\left(\mathbf{S}_{0}\right)-\mathbf{f}(\mathbf{S}) \geq \mathbf{0}, \quad \mathbf{f}_{0 v}\left(\mathbf{S}_{0}\right)-\mathbf{f}_{v}\left(\mathbf{S}_{\mathbf{v}}\right) \geq \mathbf{0}, \quad\left[A_{s}\right] \mathbf{S}+\left[A_{v}\right] \mathbf{S}_{\mathbf{v}}=\mathbf{F} .
$$

Она позволяет определить векторы усилий $\mathbf{S}$ и $\mathbf{S}_{v}$. Методом множителей Лагранжа она преобразуется в задачу определения седловой точки функционала

$$
\begin{aligned}
& \Pi_{R}=\frac{1}{2} \mathbf{S}^{T}[D] \mathbf{S}+\mathbf{S}^{T} \Theta_{0}-\mathbf{S}^{T}\left[A_{0 s}\right]^{T} \mathbf{u}_{0}+\lambda^{T}\left\{\mathbf{f}(\mathbf{S})-\mathbf{f}_{0}\left(\mathbf{S}_{0}\right)\right\}+\lambda_{v}^{T}\left\{\mathbf{f}_{v}\left(\mathbf{S}_{v}\right)-\mathbf{f}_{0 v}\left(\mathbf{S}_{0}\right)\right\}+ \\
& \mathbf{u}^{T}\left\{\mathbf{F}-\left[A_{s}\right] \mathbf{S}-\left[A_{v}\right] \mathbf{S}_{v}\right\} ; \quad \lambda \geq 0, \quad \lambda_{v} \geq 0 .
\end{aligned}
$$

Условие стационарности этого функционала по всем переменным представляет систему уравнений (49). Принимая, что условие стационарности по переменным $\mathbf{S}$ и $\mathbf{S}_{v}$ выполняется в качестве предварительного условия, получается математическая модель кинематической постановки задачи:

$$
\mathbf{u}^{T} \mathbf{F}-\frac{1}{2} \mathbf{S}^{T}[D] \mathbf{S}-\lambda^{T}[f(\mathbf{S})] \mathbf{S}-\lambda_{v}^{T}\left[f_{v}\left(\mathbf{S}_{v}\right)\right] \mathbf{S}_{v}+\lambda^{T}\left\{\mathbf{f}(\mathbf{S})-\mathbf{f}_{0}\left(\mathbf{S}_{0}\right)\right\}+\lambda_{v}^{T}\left\{\mathbf{f}_{v}\left(\mathbf{S}_{v}\right)-\mathbf{f}_{0 v}\left(\mathbf{S}_{0}\right)\right\} \rightarrow \max
$$

при условиях

$$
\begin{aligned}
& {[D] \mathbf{S}+[f(\mathbf{S})]^{T} \lambda-\left[A_{s}\right]^{T} \mathbf{u}=\left[A_{0 s}\right]^{T} \mathbf{u}_{0}-\Theta_{0},} \\
& {\left[f_{v}\left(\mathbf{S}_{v}\right)\right]^{T} \lambda_{v}-\left[A_{v}\right]^{T} \mathbf{u}=\mathbf{0}, \quad \lambda \geq 0, \quad \lambda_{v} \geq \mathbf{0} .}
\end{aligned}
$$


Неизвестными в ней являются векторы усилий $\mathbf{S}$ и $\mathbf{S}_{v}$, векторы пластических множителей $\lambda . \lambda_{v}$ и вектор перемещений и .

Двойственная пара равновесных математических моделей задачи расчета остаточных усилий и перемещений имеет такой вид:

а) статическая постановка -

$$
\frac{1}{2} \mathbf{S}_{r}^{T}[D] \mathbf{S}_{r} \rightarrow \min
$$

при условиях

$$
\mathbf{f}_{0}\left(\mathbf{S}_{0}\right)-\mathbf{f}\left(\mathbf{S}_{e}+\mathbf{S}_{r}\right) \geq \mathbf{0}, \quad \mathbf{f}_{0 v}\left(\mathbf{S}_{0}\right)-\mathbf{f}_{v}\left(\mathbf{S}_{e v}+\mathbf{S}_{r v}\right) \geq \mathbf{0}, \quad\left[A_{s}\right] \mathbf{S}_{r}+\left[A_{v}\right] \mathbf{S}_{r v}=\mathbf{0}
$$

б) кинематическая постановка -

$$
\begin{aligned}
& -\frac{1}{2} \mathbf{S}_{r}^{T}[D] \mathbf{S}_{r}-\lambda^{T}\left[f\left(\mathbf{S}_{e}+\mathbf{S}_{r}\right)\right] \mathbf{S}_{r}-\lambda_{v}^{T}\left[f_{v}\left(\mathbf{S}_{e v}+\mathbf{S}_{r v}\right)\right] \mathbf{S}_{r v}+ \\
& +\lambda^{T}\left\{\mathbf{f}\left(\mathbf{S}_{e}+\mathbf{S}_{r}\right)-\mathbf{f}_{0}\left(\mathbf{S}_{0}\right)\right\}+\lambda_{v}^{T}\left\{\mathbf{f}_{v}\left(\mathbf{S}_{e v}+\mathbf{S}_{r v}\right)-\mathbf{f}_{0 v}\left(\mathbf{S}_{0}\right)\right\} \rightarrow \max
\end{aligned}
$$

при условиях

$$
\begin{aligned}
& {[D] \mathbf{S}_{r}+\left[f\left(\mathbf{S}_{e}+\mathbf{S}_{r}\right)\right]^{T} \lambda-\left[A_{s}\right]^{T} \mathbf{u}_{r}=\mathbf{0}, \quad \lambda \geq \mathbf{0},} \\
& {\left[\int_{v}\left(\mathbf{S}_{e v}+\mathbf{S}_{r v}\right)\right]^{T} \lambda_{v}-\left[A_{v}\right]^{T} \mathbf{u}_{r}=\mathbf{0}, \quad \lambda_{v} \geq \mathbf{0} .}
\end{aligned}
$$

В случае линейных условий текучести

$$
[\Phi] \mathbf{S} \leq[\rho] \mathbf{S}_{0}, \quad\left[\Phi_{v}\right] \mathbf{S}_{v} \leq\left[\rho_{v}\right] \mathbf{S}_{0}
$$

кинематические постановки задач можно значительно упростить. Во-первых, используя геометрические уравнения, можно исключить усилия

$$
\mathbf{S}_{r}=[D]^{-1}\left\{\left[A_{s}\right]^{T} \mathbf{u}_{r}-[\Phi]^{T} \lambda\right\} .
$$

Тогда получается задача

$$
\begin{aligned}
& -\frac{1}{2} \mathbf{u}_{r}^{T}[K] \mathbf{u}_{r}-\frac{1}{2} \lambda^{T}[\Phi][D]^{-1}[\Phi]^{T} \lambda-\mathbf{u}_{r}^{T}\left[A_{s}\right][D]^{-1}[\Phi]^{T} \lambda+ \\
& +\lambda^{T}\left\{[\Phi] \mathbf{S}_{e}-[\rho] \mathbf{S}_{0}\right\}+\lambda_{\nu}^{T}\left\{\left[\Phi_{\nu}\right] \mathbf{S}_{e v}-\left[\rho_{\nu}\right] \mathbf{S}_{0}\right\} \rightarrow \max ,
\end{aligned}
$$

при условиях

$$
\left[\Phi_{v}\right]^{T} \lambda_{v}-\left[A_{v}\right]^{T} \mathbf{u}_{r}=\mathbf{0}, \quad \lambda \geq 0, \quad \lambda_{v} \geq 0
$$


Кроме того могут быть исключены линейно зависимые перемешения. Для этого предварительн необходимо опрељелить репение уравнений совместности переменений межлу элементами в видс:

$$
\mathbf{u}=[L]\left[\Phi_{v}\right]^{T} \lambda_{v}+\left[L_{1}\right] \mathbf{u}_{1} \quad \text { илии } \quad \mathbf{u}_{r}=[L]\left[\Phi_{v}\right]^{T} \lambda_{v}+\left[I_{1}\right] \mathbf{u}_{r 1}
$$

Здесь

$$
[L]=\left[\Gamma^{\prime}\right]\left[\begin{array}{c}
\left(\left[A_{v 2}\right]^{T}\right)^{-1} \\
{[0]}
\end{array}\right] ; \quad\left[I_{11}\right]=[\Gamma]\left[\begin{array}{c}
-\left(\left[A_{v 2}\right]^{T}\right)^{-1}\left[A_{v \cdot 1}\right]^{T} \\
{[E]}
\end{array}\right] ;
$$

$\left[A_{\nu_{2}}\right]^{T}-$ базисная матрица геометрических уравнений конечных элементов, отвечающая базису $\mathbf{u}_{r 2}$ или $\mathbf{u}_{2} ;[\mathrm{I}]$ - матрипа преобразования, определяюпая связь $\mathbf{u}_{r}=[\Gamma] \tilde{\mathbf{u}}_{r}$, где $\widetilde{\mathbf{u}}_{r} \equiv\left\{\mathbf{u}_{r 2}, \mathbf{u}_{r 1}\right\}^{T} ;[0],[E]-$ нулевая и единичная матрицы. Тогда, например, задача (66) с учетом (68) и (70) преобразуетея к следуюпей задаче квадратичного гтрограммирования:

$$
\begin{gathered}
-\frac{1}{2} \mathbf{u}_{r 1}^{T}\left[\Pi_{u}\right] \mathbf{u}_{r 1}-\frac{1}{2} \lambda^{T}\left[\Phi_{\lambda}\right] \lambda-\frac{1}{2} \lambda_{v}^{T}\left[\Pi_{v}\right] \lambda_{v}-\mathbf{u}_{r 1}^{T}\left[\Pi_{u v}\right] \lambda_{v}+\lambda_{v}^{T}\left[\Pi_{\lambda}\right] \lambda+\mathbf{u}_{r 1}^{T}\left[\Pi_{u \lambda}\right] \lambda+ \\
+\lambda^{T}\left\{[\Phi] \mathbf{S}_{e}-[\rho] \mathbf{S}_{0}\right\}+\lambda_{v}^{T}\left\{\left[\Phi_{v}\right] \mathbf{S}_{\epsilon_{v}}-\left[\rho_{v}\right] \mathbf{S}_{0}\right\} \rightarrow \mathbf{m a x} \\
\lambda \geq \mathbf{0}, \quad \lambda_{v} \geq \mathbf{0 .}
\end{gathered}
$$

Здесь

$$
\begin{aligned}
& {\left[\Phi_{\lambda}\right]=[\Phi][D]^{-1}[\Phi]^{T}, \quad\left[\Pi_{v}\right]=\left[\Phi_{\nu}\right][L]^{T}[K][L]\left[\Phi_{\nu}\right]^{T},} \\
& {\left[\Pi_{u}\right]=\left[L_{1}\right]^{T}[K]\left[L_{1}\right], \quad\left[\Pi_{u \nu}\right]=\left[L_{1}\right]^{T}[K][L]\left[\Phi_{\nu}\right]^{T},} \\
& {\left[\Pi_{\lambda}\right]=\left[\Phi_{v}\right][L]^{T}\left[A_{s}\right][D]^{-1}[\Phi]^{T}, \quad\left[\Pi_{u \lambda}\right]=\left[L_{1}\right]^{T}\left[A_{s}\right][D]^{-1}[\Phi]^{T} .}
\end{aligned}
$$

Во--вторых, оттимальные ренгеня задач (64), (66) удовлетворяккт не только геометрическим уравнениям, но и уравнениям равновесия. Поэтому уравнения равновесия заранее можно присоединить к ограничениям этих залач. Тогда, исліользуя решение системы уравнений (50) или (59), и'з числа неизнсстных можно исключить все усиция и перемешения. Нсизвестными остаются только ограниченныс 1 жо заку пластические множители и задача (66) сводится к залаче безусловной максимизации:

$$
\begin{aligned}
& -\frac{1}{2} \lambda^{T}[C] \lambda-\frac{1}{2} \lambda_{\nu}^{T}\left[C_{v}\right] \lambda_{v}-\lambda_{\nu}^{T}\left[C_{\lambda}\right] \lambda+\lambda^{T}\left\{[\Phi] \mathbf{S}_{r}-[\rho] \mathbf{S}_{0}\right\}+\lambda_{\nu}^{T}\left\{\left[\Phi_{v}\right] \mathbf{S}_{c \nu}-\left[\rho_{v}\right] \mathbf{S}_{0}\right\} \rightarrow \max , \\
& \lambda \geq 0, \lambda_{v} \geq 0 .
\end{aligned}
$$


Здесь

$[C]=[\Phi][G]^{T}[D][G][\Phi]^{T}, \quad\left[C_{v}\right]=\left[\Phi_{v}\right]\left[G_{v}\right]^{T}[D]\left[G_{v}\right]\left[\Phi_{v}\right]^{T}, \quad\left[C_{\lambda}\right]=\left[\Phi_{v}\right]\left[G_{v}\right]^{T}[D][G][\Phi]^{T}$.

Неизвестными в этой задаче являются лишь пластические множители. Поэтому отыскание ее решения проще.

Аналогичным образом может быть преобразована и математическая модель (64). Однако при этом получается более сложная по сравнению с (72) задача. Поэтому для определения пшастических множителей целесообразно решить задачу (72). Тогда пластические деформации

$$
\Theta_{p}=[\Phi]^{T} \lambda, \quad \Theta_{p v}=\left[\Phi_{v}\right]^{T} \lambda_{v} .
$$

А для расчета усилий и перемещений целесообразно применять зависимости (51)(56) или (60)-(62). Такой алгоригм решения задачи анализа НДС упругопластической конструкции проще по сравнению с прямым решением двойственной пары задач (63), (64) или пары (65), (66). Возможность построения математической модели (72) появляется лишь в том случае, когда уравнения равновесия и геометрические уравнения строятся с соблюдением двойственных связей. Это показывает, что формулировка задач анализа упругопластических конструкций с соблюдением двойственных связей позволяет угростить алгоритм решения этих задач.

\section{4. Учет разрывов в задачах анализа НДС при повторных нагружениях}

В работах [2, 3] и других приведены двойственные математические модели задач анализа и оптимизации упругопластических конструкций, в том числе пластинок, при повторно-переменных нагружениях. Однако возможность образования разрывов полей перемещений и линейных пластических шарниров (линий разрушения) в них не учитывается. Вышеизложенная методика позволяет это сделать. Учет возможных разрывов перемещений в двойственных математических моделях задач анализа и оптимизации при повторных нагружениях ниже иллюстрируется на примере задачи анализа остаточных усилий.

Статическая постановка задачи:

$$
\frac{1}{2} \mathbf{S}_{r}^{T}[D] \mathbf{S}_{r} \rightarrow \min
$$

при условиях

$$
\begin{gathered}
\mathbf{f}_{0}\left(\mathbf{S}_{0}\right)-\mathbf{f}\left(\mathbf{S}_{r}+\mathbf{S}_{e j, \text { max }}\right) \geq \mathbf{0}, \quad \mathbf{f}_{0 v}\left(\mathbf{S}_{0}\right)-\mathbf{f}_{v}\left(\mathbf{S}_{r v}+\mathbf{S}_{e v j, \max }\right) \geq \mathbf{0}, \\
\mathbf{f}_{0}\left(\mathbf{S}_{0}\right)-\mathbf{f}\left(\mathbf{S}_{r}+\mathbf{S}_{e j, \text { min }}\right) \geq \mathbf{0}, \quad \mathbf{f}_{0 v}\left(\mathbf{S}_{0}\right)-\mathbf{f}_{v}\left(\mathbf{S}_{r v}+\mathbf{S}_{e v j \text { min }}\right) \geq \mathbf{0}, \\
{\left[A_{s}\right] \mathbf{S}_{r}+\left[A_{v}\right] \mathbf{S}_{r v}=\mathbf{0} ; \quad j=1.2, \ldots \rho .}
\end{gathered}
$$


Здесь $\mathbf{S}_{e j, \max }, \mathbf{S}_{e j, \min }$ - векторы заданных экстремальных усилий, соответствующие $\boldsymbol{j}-$ й паре симметричных вершин годографа упругих усилий [3]: $p$ - число пар симметричныгх вершин этого тодографа.

Кинематическая постановка задачи имеет такой вид:

$$
\left\{\begin{array}{l}
-\frac{1}{2} \mathbf{S}_{r}^{T}[D] \mathbf{S}_{r}-\sum_{j} \lambda_{j, \max }^{T}\left[f\left(\mathbf{S}_{r}+\mathbf{S}_{e j, \max }\right)\right] \mathbf{S}_{r}-\sum_{j} \lambda_{j, \min }^{T}\left[f\left(\mathbf{S}_{r}+\mathbf{S}_{e j, \min }\right)\right] \mathbf{S}_{r}- \\
-\sum_{j} \lambda_{v j, \max }^{T}\left[f_{v}\left(\mathbf{S}_{r v}+\mathbf{S}_{e v, \max }\right)\right] \mathbf{S}_{r v}-\sum_{j} \lambda_{v j, \min }^{T}\left[f_{v}\left(\mathbf{S}_{r v}+\mathbf{S}_{e v, \min }\right)\right] \mathbf{S}_{r v}+ \\
+\sum_{j} \lambda_{j, \max }^{T}\left\{\mathbf{f}\left(\mathbf{S}_{r}+\mathbf{S}_{e j, \max }\right)-\mathbf{f}_{0}\left(\mathbf{S}_{0}\right)\right\}+\sum_{j} \lambda_{j, \min }^{T}\left\{\mathbf{f}\left(\mathbf{S}_{r}+\mathbf{S}_{e j, \min }\right)-\mathbf{f}_{0}\left(\mathbf{S}_{0}\right)\right\}+ \\
+\sum_{j} \lambda_{v j, \max }^{T}\left\{\mathbf{f}_{v}\left(\mathbf{S}_{r v}+\mathbf{S}_{e v j, \max }\right)-\mathbf{f}_{0 v}\left(\mathbf{S}_{0}\right)\right\}+\sum_{j} \lambda_{v j, \min }^{T}\left\{\mathbf{f}_{v}\left(\mathbf{S}_{r v}+\mathbf{S}_{e v j, \min }\right)-\mathbf{f}_{0 v}\left(\mathbf{S}_{0}\right)\right\}
\end{array}\right\} \rightarrow \max
$$

при услтовиях

$$
\begin{aligned}
& {[D] \mathbf{S}_{r}+\sum_{j}\left[f\left(\mathbf{S}_{r}+\mathbf{S}_{e j, \max }\right)\right]^{T} \lambda_{j, \max }+\sum_{j}\left[f\left(\mathbf{S}_{r}+\mathbf{S}_{e j, \min }\right)\right]^{T} \lambda_{j, \min }-\left[A_{s}\right]^{T} \mathbf{u}_{r}=\mathbf{0},} \\
& \sum_{j}\left[f_{v}\left(\mathbf{S}_{r v}+\mathbf{S}_{e j, \max }\right)\right]^{T} \lambda_{v j, \max }+\sum_{j}\left[f_{v}\left(\mathbf{S}_{r v}+\mathbf{S}_{e j, \min }\right)\right]^{T} \lambda_{v j, \min }-\left[A_{v}\right]^{T} \mathbf{u}_{r}=0, \\
& \lambda_{j, \max } \geq 0, \quad \lambda_{j, \min } \geq 0, \quad \lambda_{v j, \max } \geq 0, \quad \lambda_{v j, \min } \geq 0 ; \quad j=1,2, \ldots, \rho .
\end{aligned}
$$

Четвертый и пятый члены функции цели этой задачи выражают энергию диссипации в повехностях разрыва перемещений. Второе уравнение в ограничениях задачи представляет собой геометрические уравнения поверхностей раздела конечных элементов. Если пластические деформации в них отсутствуют, то они выражают требование непрерывности перемещений.

\section{5. Заключение}

Для конечноэлементных моделей упругопластических конструкций представлены двойственные пары обцих математических моделей задач анализа суммарных, а также остаточных усилий и перемещений при разных видах нагружения. В них предполагается возможность разрывов полей перемещений и учитывается диссипация энергии в местах этих разрывов. На основе аппроксимаций полей усилий или напряжений разработаны дискретные выражения уравнений равновесия, геометрических уравнений и условий текучести. Показано, что для дискретизации условий текучести могут применяться классические методы коллокаций: точечная коллокация, коллокация в подобласти и метод Бубнова-Галеркина. Последний из 
них приводит $\mathbf{k}$ наилупшей аппроксимации условий текучести и пластических деформаций, а заодно и диссипативной энергии. Разработана общая для геометрически линейных задач упругопластических конструкций методика дискретизации уравнений равновесия и условий текучести.

Построена полная система уравнений и двойственные пары дискретных равновесных математических моделей статической и кинематической постановки задач анализа параметров НДС. Для их построения применяются равновесные конечные элементы и метод множителей Лагранжа. Математические модели обеспечивают непрерывность полей перемещений в тех местах раздела конечных элементов, где пластические деформации и трещины отсутствуют. Похазано, что формулировка задач анализа упрутопластических конструкций с соблюдением двойственных связей позволяет упростить алгоритм решения этих задач. Проиллюстрирован также учет разрывов полей перемещений в задачах анализа НДС при повторных нагружениях.

Построенные равновесные математические модели задач позволяют определить верхние значения параметров НДС конструкций. Хотя исследования касаются тонкостенных конструкций, однако их дискретные математические модели общие и распространяются как на стержневые системы, так и на трехмерные тела.

\section{Литература}

1. Дж. Майер. Квадратичное программирование и теория упруго-идеальнопластических конструкций // Механика (ИЛ), 1969, №. 6 (118), с. 112-128.

2. А.А Чирас. Математические модели анализа и оптимизации упругопластических систем. Вильнюс: Мокслас, 1982. 112 с.

3. Ю.Ю. Аткочюнас. Расчет упругопластических систем при повторных нагружениях Виљнюс: Изд-во науки и энциклопедий, 1994. 150 с.

4. А.А. Данюнас. Метод равновесных конечных элементов в задачах анализа и оптимизации упругопластических пластин // Прикладная механика и оптимизация (Литовский механический сборник, №.27), Вильнюс, 1985, с. 28-39.

5. С. Каланта. Двойственные постановки задач предельного равновесия для конечно- элементных моделей // 4 -sios tarptautinès konferencijos "Naujos statybinès medžiagios, konstrukcijos ir technologijos" straipsniai, III t. Vilnius: Technika, 1995, p. 160165 .

6. С.А. Каланта. Двойственные математические модели задач оптимизации жестко- пластических систем конечных элементов // Строит. механика и расчет сооружений, No. 3, 1983, с. 11-15.

7. В. Койтер. Общие теоремы теории упругопластических сред. М.: ИЛ, 1961.78 с. 
8. Р. Галлагер. Метод конечных элементов. Основы. М.: Мир, 1984. 428 с.

9. S. Kalanta. Takumo sąlygos baigtinių elementų modeliams // 4-sios tarptautinès konferencijos "Naujos statybinès medžiagios, konstrukcijos ir technologijos" straipsniai, III t. Vilnius: Technika, 1995, p. 260-265.

10. О. Зенкевич. Метод конечных элементов в технике. М.: Мир, 1975. 541 с.

11. Nguen Dang H. Direct limit analysis via rigid-plastic finite elements // Comput. Meth. Appl. Mech. Eng., vol. 8, No 1, 1976, p. 81-116.

12.А Чирас, С.А Каланта. Определение матрицы функции текучести конечного элемента. Вильнюс, 1977, 6 с. Деп. в ЛитИИ 28.04.77, No. $196-77$.

\section{TAMPRIŲ-PLASTIŠKŲ KONSTRUKCIJU SU TRŪKIOMIS POSLINKIŲ FUNKCIJOMIS ANALIZĖS UŽDAVINIŲ DUALŪS MATEMATINIAI MODELIAI}

\section{S. Kalanta}

S a n tra u k a

Nagrinejjami idealiai tamprių-plastiškų konstrukcijų, veikiamy̨ j̨vairių išorinių poveikių (apkrova, išankstinis deformavimas, atramy sèdimas), įtempto-deformuoto bũvio analizès uždaviniai. Remiantis ekstreminiais energetiniais principais ir dualumo teorija sudaryti bendri ir diskretiniai dualüs šį uždavinių matematiniai modeliai. Jų naujumas - juertinami galimi poslinkių funkciju trūkiai ir energijos disipacija plastiniuose paviršiuose tarp baigtiniu elementų. Pagrindiniu priklausomybiu (statikos ir geometrinių lygčiu, takumo sąlygų) diskretinès išraiškos ir diskretiniai matematiniai modeliai sudaryti panaudojant pusiausviru baigtinių elementy̨ metodą ir yra pusiausvirūs. Jie leidžia nustatyti ịrąžų (ịtempimy̨) ir poslinkių viršutines reikšmes. Parodyta, kad iš visu klasikinių kolokacijos metodų, kurie gali būti naudojami takumo sąlygu diskretizacijai, tiksliausiai plastinès deformacijos ir energijos disipacija modeliuojamos diskretizuojant takumo sąlygas Bubnovo-Galiorkino metodu.

\section{DUAL MATHEMATICAL MODELS OF ELASTOPLASTIC STRUCTURES ANALYSIS PROBLEM ESTIMATING DISCONTINUITY OF DISPLACEMENTS}

\section{S. Kalanta}

Su m mary

A problem of ideal elastoplastic structures stress-strain field determination is considered in the article. The general dual mathematical models (static and kinematic formulation) of analysis problem is derived on the basis of the extremal energy principles and theory of duality. The different external effects are estimated, 
namely: load, initial strains, prestressing and support settlements. At first, on the basis of the complementary energy principle the mathematical model of static formulation of the problem is made. The kinematic formulation of the problem is obtained on the basis of Lagrange's multipliers method; this corresponds to the minimum total energy principle for a kinematically admissible displacements. In these mathematical models the possible discontinuity of displacements and the dissipation of energy in the place of those discontinuities are estimated what was not done in the previous publications.

The discrete expressions of fundamental relationships (equilibrium and geometric equations, yield conditions) and a dual pair of discrete equilibrium mathematical models are obtained on the basis of general static formulation of the problem using equilibrium finite elements. They permit to determine the upper meanings of the stress and displacements of structures. In the article it has been shown, that the approximation of yield conditions by Bubnov-Galiorkin's collocation method gives the more accurate results. 